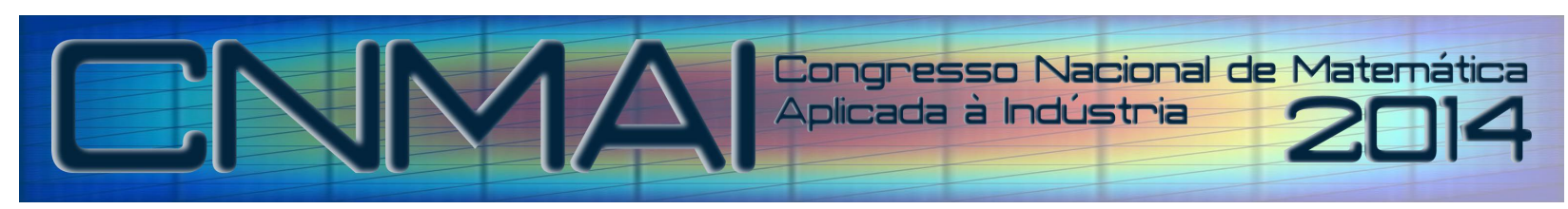

18 a 21 de novembro de 2014, Caldas Novas - Goiás

\title{
ESTUDO DE UM MODELO DE PROGRAMAÇÃO INTEIRA PARA O PROBLEMA DE LOCALIZAÇÃO DE ESTAÇÕES E AMBULÂNCIAS
}

\author{
Raina Ribeiro Marques, raina-marques@hotmail.com \\ Thiago Alves de Queiroz, taq@ufg.br
}

Universidade Federal de Goiás / Regional Catalão, Departamento de Matemática. Av. Dr. Lamartine Pinto de Avelar, 1120, Setor Universitário, 75704-020, Catalão-GO, Brasil.

\begin{abstract}
Resumo: O problema de localização de facilidades aparece em aplicações do setor público e privado, como na determinação de postos policiais, hospitais, escolas, fábricas, depósitos, etc. Este trabalho investiga um modelo de programação inteira cujo objetivo é minimizar o custo de instalação de ambulâncias e pontos de atendimento (estações) de forma a garantir um nível mínimo de confiabilidade do serviço prestado. $O$ modelo investigado foi codificado na linguagem $C$ e resolvido pelo algoritmo Branch-and-Cut disponível nas bibliotecas do pacote GUROBI OPTIMIZER ${ }^{\circledR}$ e ILOG CPLEX ${ }^{\circledR}$. A partir dos resultados, foram realizadas análises e comparações com outros trabalhos da literatura que apresentavam modelos similares. Os resultados obtidos com a implementação feita permitiram melhorar o resultado dos outros modelos da literatura em até 16,7\%. Também foram realizadas comparações quanto ao tempo de execução dos pacotes de otimização.
\end{abstract}

Palavras-chave: Localização de facilidades, nível de confiabilidade, programação inteira.

\section{INTRODUÇÃO}

Uma importante área de pesquisa da Pesquisa Operacional envolve a aplicação de técnicas de resolução dos problemas de localização de facilidades. Estes problemas tratam a questão de onde instalar um objeto (ou objetos), chamados facilidades e que podem ser fábricas, depósitos, escolas, etc. Uma facilidade deverá interagir com clientes, que podem ser depósitos, unidades de vendas, estudantes, e podem ter localizações fixas. Alguns casos associam informações de demandas e capacidade de serviços (ARENALES et al. 2007).

A teoria de localização obteve um maior interesse com a publicação de Hakimi em meados da década de 1960, cujo objetivo era determinar a localização de postos de serviços em redes (HAKIMI, 1964) e postos policiais (HAKIMI, 1965). Desde então, a teoria da localização tem se desenvolvido. Alguns dos requisitos dos problemas de localização de facilidades consistem em determinar onde as instalações devem ser localizadas, quais fornecedores devem ser utilizados, quantos centros de distribuição à empresa devem operar e onde eles devem estar localizados, que clientes devem ser supridos, que linhas de produtos devem ser produzidas e que tipos de transportes utilizar.

Uma das importantes aplicações referente à teoria da localização é a localização de postos de saúde, corpos de bombeiro, hospitais, Serviço de Atendimento Móvel de Saúde (SAMU), dentre outros. Devido a essa demanda, em Minas Gerais, para a cidade de Belo Horizonte, foi desenvolvido um modelo de programação linear inteira a fim de realocar as bases de operação para reduzir o tempo de resposta do SAMU e também diminuir o número dessas bases, contudo garantindo sempre o melhor atendimento para toda a população e satisfazendo as demandas impostas (NOGUEIRA, 2011).

Outra aplicação que se pode citar é a da localização de aeroportos. Uma empresa na região sudeste do Brasil do ramo de fármacos decidiu ampliar a sua planta devido a uma maior demanda de suas mercadorias. Porém, a empresa optou por fazer a distribuição de seus produtos por via aérea, pois era inviável por via terrestre em consequência do alto valor agregado. Com isso, foi utilizada a teoria da localização, planejando um modelo capaz de definir o melhor aeroporto com infraestrutura capaz de atender a distribuição dos produtos, com custos logísticos globais mínimos (SILVA; SUCENA, 2009).

Neste trabalho é abordado o problema de localizar pontos de atendimento e ambulâncias necessárias para atender uma dada demanda, com custo mínimo de implantações e garantindo um nível mínimo de confiança, seguindo o trabalho de Mohaymany et al. (2012). Esta pesquisa visa proporcionar economia na instalação das estações e ambulâncias, assim como, melhorar o atendimento a população, oferecendo atendimentos mais ágeis para as pessoas 
necessitadas. Além disso, a definição do número de ambulâncias para cada demanda abona a falta de ambulâncias, podendo ser crucial para salvar vidas.

A literatura sobre a programação linear na área da saúde é vasta. Autores como Owen e Daskin (1998), Goldberg (2004), Galvão e Morabito (2008), Revelle et al. (2008) e Mohaymany et al. (2012) investigaram o problema de localização dos serviços de emergência médica. As estratégias utilizadas foram baseadas em dois modelos: Problema de Localização de Conjunto de Cobertura (PLCC); e Problema de Localização de Cobertura Máxima (PLCM), introduzidos por Toregas et al. (1971) e Church e Revelle (1974), respectivamente. O objetivo do modelo PLCC é minimizar o número de ambulâncias necessárias para cobrir todos os pontos de demanda, considerando um tempo de cobertura específico ou distância de chegada. Já o modelo PLCM maximiza a cobertura de demanda sujeita a um número limite de ambulâncias e/ou estações (pontos de atendimento). Porém, devido a restrições práticas como ambulâncias ocupadas devido ao despacho para outras chamadas e trabalho associado à manutenção, esses modelos não oferecem um bom desempenho.

Por outro lado, alguns pesquisadores resolveram trabalhar com modelos de coberturas múltiplas. Tais modelos podem ser encontrados em Daskin e Stern (1981), Hogan e Revelle (1986), Pirkull e Schilling (1988), Batta e Marmur (1990), Narasimham et al. (1992) e Gendreau et al. (1997). Porém, os modelos propostos possuem dois problemas: maximizar a cobertura múltipla apenas para um conjunto limitado de pontos de demanda, seguindo o objetivo do modelo PLCM; e, eles tendem a fornecer uma cobertura múltipla para os pontos com maiores exigências, sem qualquer limite ou limite razoável para garantir um nível específico de disponibilidade da ambulância. Assim, autores foram incentivados a propor novos modelos, usando outros tipos de estratégicas como: modelos probabilísticos, teorias das filas, simulação e programação matemática.

Diante disto, este trabalho investiga o modelo proposto por Mohaymany et al. (2012) para o qual foi proposta uma simples modificação, permitindo agilizar a obtenção da solução ótima. A Seção 2 apresenta o modelo desses autores e traz a modificação proposta. Os resultados computacionais são apresentados na Seção 3, incluindo a comparação de dois solvers comerciais aplicados sobre o modelo em estudo. Conclusões e direções para trabalhos futuros são apresentadas na Seção 4 .

\section{MODELO INVESTIGADO}

O modelo investigado, proposto por Mohaymany et al. (2012), consiste no UBUL (Upper-Bound Unavailability Location). Ele ataca um problema de localização de cobertura completa com o objetivo de determinar o menor número de ambulâncias e estações necessárias para cobrir totalmente uma determinada demanda.

O modelo trabalha com a garantia de que a população seja atendida de acordo com um nível de confiabilidade estabelecido. Consequentemente, o nível de confiabilidade influencia na quantidade de instalações que são necessárias para atender a demanda. O modelo também apresenta uma restrição relacionada à indisponibilidade das ambulâncias, diferentemente de outros trabalhos da literatura. Tal modelo proporciona uma melhor solução para o problema, pois leva em consideração o tempo médio gasto para atender as solicitações das demandas e a fração máxima de ocupação das ambulâncias, ambas melhores especificadas adiante.

Estudo semelhante a este pode ser encontrado nos trabalhos de Borras (2000) e Borras e Pastor (2002), cujos modelos investigados foram utilizados para a comparação dos resultados aqui obtidos. Segue a formulação completa por programação inteira do modelo UBUL.

Conjuntos e índices:

- $\quad I$ : Conjunto de pontos de demanda ou clientes (elementos de $I$ serão denominados por $i$ ).

- $\quad J$ : Conjunto de estações (elementos de $J$ serão denominados por $j$ ).

- $A$ : Conjunto de ambulâncias (elementos de $A$ denominados por $a$ ).

- $A_{j}$ : Conjunto de ambulâncias que podem ser localizadas na estação $j$, onde $A_{j}$ é subconjunto de $A$.

- $\quad N_{i}$ : Conjunto de ambulâncias que atendem o ponto de demanda $i$.

- $\quad B_{a}$ : Conjunto de pontos de demanda atendidos pela ambulância $a$, isto é, que estão no raio $S$ de cobertura da ambulância.

Dados de entrada:

- $\quad q_{i}$ : Tempo médio gasto (em horas/dia) para atender as solicitações da demanda $i$.

- $\quad S$ : Distância de cobertura para uma ambulância $a$ localizada em qualquer estação $j$.

- $\quad \alpha$ : Nível de confiabilidade mínimo para assegurar a cobertura especificada.

- $\quad f$ : Número mínimo de ambulâncias necessário para cobrir cada ponto de demanda dado o nível mínimo de confiabilidade.

- $\quad \rho$ : Fração máxima de ocupação imposta a cada ambulância. Obtida da equação $\rho=\sqrt[f]{(1-\alpha)}-0,01$, (REVELLE; HOGAN (1989) apud MOHAYMANY et al. 2012).

- $\quad L$ : Número máximo de ambulâncias que podem atender certo ponto de demanda $i: L=\operatorname{Max}\left\{\left|N_{i}\right|\right\}$, para $\forall i \in I$.

- $\quad \beta$ : Custo de implantação das ambulâncias. Assume-se que o custo é igual para toda ambulância. 
- $\quad \gamma$ : Custo de implantação das estações. Assume-se o mesmo custo, independentemente do local ou da estação.

- M: Número inteiro grande.

Formulação do modelo:

- $x_{a}=\left\{\begin{array}{l}1, \text { se ambulância a é implantada. } \\ 0, \text { caso contrário. }\end{array}\right.$

- $\quad z_{j}=\left\{\begin{array}{l}1, \text { se no mínimo } 1 \text { ambulância é posicionada na estação } j . \\ 0, \text { caso contrário. }\end{array}\right.$

- $y_{i k}=\left\{\begin{array}{l}1, \text { se o ponto de demanda } i \text { é coberto por exatamente } k \text { ambulâncias. } \\ 0, \text { caso contrário. }\end{array}\right.$

$$
\text { Minimizar } Z=\beta \sum_{a \in A} x_{a}+\gamma \sum_{j \in J} z_{j}
$$

Sujeito a:

$$
\begin{array}{ll}
24 \rho \geq \sum_{i \in B_{a}}\left(q_{i} \sum_{k=1}^{L} y_{i k} \frac{1}{k}\right)+\left(x_{a}-1\right) M, & \forall a \in A \\
\sum_{a \in N_{i}} x_{a}=\sum_{k=1}^{L} y_{i k} k, & \forall i \in I \\
\sum_{k=1}^{L} y_{i k}=1, & \forall i \in I \\
\sum_{a \in N_{i}} x_{a} \geq f, & \forall i \in I \\
M z_{j} \geq \sum_{a \in A_{j}} x_{a}, & \forall j \in J \\
x_{a}, z_{j}, y_{i k} \in\{0,1\} & \forall a \in A, \forall j \in J, \forall i \in I, k=1,2, \ldots, L
\end{array}
$$

A função objetivo minimiza o custo total relacionado à instalação das ambulâncias e estações. A restrição (1) é relacionada à indisponibilidade da ambulância de tal forma que a demanda não deve exceder sua capacidade, ou seja, para toda ambulância $a$, a fração máxima de ocupação por dia deve ser maior ou igual ao tempo médio gasto para atender as solicitações dos pontos de demanda $i$, atendidas por $a$, caso $a$ esteja realmente instalada. As restrições (2) e (3) introduzem a variável $y_{i k}$ no modelo de modo que $y_{i k}$ será 1 se e somente se o ponto de demanda $i$ for coberto por exatamente $k$ ambulâncias. A restrição (4) exige que o ponto de demanda $i$ seja coberto por no mínimo $f$ ambulâncias. A restrição (5) garante que as ambulâncias implantadas devem ser posicionadas em estações abertas. Por fim, a restrição (6) impõe que as variáveis $x_{a}, z_{j}$ e $y_{i k}$ sejam inteiras (mais especificamente, binárias).

Observa-se que o parâmetro $L$, número máximo de ambulâncias que podem atender uma determinada demanda, é obtido observando todos os pontos de demanda $i$. As restrições e as variáveis $y_{i k}$ usam $L$ como sendo um valor fixo. Porém, ao usar $L$ como constante, ocorre um acréscimo desnecessário de variáveis do tipo $y_{i k}$. Note que é suficiente considerar apenas o número máximo de ambulâncias que realmente atende o ponto de demanda $i$, isto é, para cada ponto $i$ existe um máximo de ambulâncias que o atende e que, não necessariamente, é o mesmo para outro ponto $i^{\prime}$. Observe que o número de ambulâncias que atende certo ponto de demanda está relacionado com a distância de cobertura $S$. Diante disto, modificou-se o modelo de forma que exista um parâmetro $L_{i}$ para cada ponto de demanda $i$. Assim, as restrições (1), (2), (3) e (6) foram rescritas de acordo com esse novo parâmetro. Por fim, tal mudança possibilita diminuir o tempo de otimização para a resolução do modelo, pois permite diminuir o número de elementos na matriz de restrições e, claramente, não afeta o valor da solução ótima. Denominou-se esta versão do modelo por UBUL-M (UBUL-Modificado).

\section{AVALIAÇÃo DO MODELO UBUL-M}




\subsection{Dados para os Testes}

Para realizar a comparação entre os resultados foram utilizados três modelos, a saber: BRLSCP (Binomial Reliability Location Set Covering Problem), que está baseado em hipóteses de que existe um tempo médio de serviço e que o número de veículos ocupados segue uma distribuição Binomial; PRLSCP (Poisson Reliability Location Set Covering Problem), em que as chamadas de emergência seguem uma distribuição de Poisson e que existe um limite superior para o tempo de serviço; e, QRLSCP (Queueing Reliability location set covering problem), com a hipótese de que as chamadas de emergência seguem uma distribuição de Poisson. Detalhes sobre estes modelos podem ser obtidos em Borras (2000) e Borras e Pastor (2002).

Considera-se para os testes uma rede de 79 nós, primeiramente introduzida por Serra (1989) e depois utilizada por Borras (2000). Existem três cenários com diferentes chamadas/dia. A distância de cobertura usada foi de $S=1,5$ milhas. Assumem-se nove níveis de confiabilidade e o custo das instalações foi definido por $\beta=1$ e $\gamma=0$. As frações de ocupação em relação ao nível de confiabilidade e ao número mínimo de ambulâncias requeridas para satisfazer determinada demanda podem ser obtidos em Mohaymany et al. (2012). Além disso, considera-se que cada chamada de emergência possui uma duração média de 45 minutos.

\subsection{Resultados}

O modelo UBUL-M foi implementado na linguagem $\mathrm{C} / \mathrm{C}++$. Usaram-se as bibliotecas do IBM ILOG CPLEX ${ }^{\circledR}$ 12.5 e do GUROBI OPTIMIZER ${ }^{\circledR}$ 5.6.3. O computador usado nos testes tem processador Intel Xeon X3430 2.40 GHz, 8 GB de memória RAM, sistema operacional Linux e foi considerada a execução em um único core. O GUROBI permitiu que o modelo fosse transcrito de forma fácil e rápida, ao contrário do CPLEX. A vantagem de ambos é fornecer um framework para codificar modelos de programação inteira e resolvê-los usando um algoritmo Branch-andCut eficiente.

$\mathrm{Na}$ Tabela 1 tem-se a quantidade de ambulâncias instaladas para os modelos UBUL-M (melhor resultado obtido ao comparar a implementação em CPLEX e GUROBI), BRLSCP, QRLSCP e PRLSCP. Os resultados obtidos ao resolver o modelo UBUL podem ser encontrados em Mohaymany et al. (2012), porém difere, para algumas instâncias, dos apresentados pelo UBUL-M devido ao tempo limite imposto, a modificação realizada no parâmetro $L i$, ou o valor de $f$ fornecido como entrada. Tais resultados foram destacados em negrito na coluna do UBUL-M.

Tabela 1: Resultados para os modelos UBUL-M, BRLSCP, QRLSCP e PRLSCP.

\begin{tabular}{|c|c|c|c|c|c|}
\hline \multicolumn{6}{|c|}{ Distância de cobertura: $S=1,5$ milhas } \\
\hline Cenários & $\alpha(\%)$ & UBUL-M & BRLSCP & QRLSCP & PRLSCP \\
\hline \multirow{9}{*}{01} & 80 & 9 & 11 & 10 & 10 \\
\hline & 82,5 & 10 & 11 & 10 & 11 \\
\hline & 85 & 12 & 12 & 11 & 12 \\
\hline & 87,5 & 12 & 13 & 13 & 13 \\
\hline & 90 & 12 & 13 & 13 & 13 \\
\hline & 92,5 & 12 & 14 & 13 & 13 \\
\hline & 95 & 12 & 14 & 14 & 14 \\
\hline & 97,5 & 12 & 14 & 17 & 17 \\
\hline & 99 & 17 & 18 & 19 & 19 \\
\hline \multirow{9}{*}{02} & 80 & 12 & 13 & 13 & 13 \\
\hline & 82,5 & 12 & 14 & 13 & 14 \\
\hline & 85 & 12 & 14 & 13 & 14 \\
\hline & 87,5 & 12 & 14 & 14 & 14 \\
\hline & 90 & 12 & 14 & 14 & 14 \\
\hline & 92,5 & 12 & 14 & 15 & 15 \\
\hline & 95 & 13 & 15 & 16 & 17 \\
\hline & 97,5 & 18 & 18 & 20 & 20 \\
\hline & 99 & 18 & 20 & 22 & 22 \\
\hline \multirow{6}{*}{03} & 80 & 18 & 18 & 18 & 20 \\
\hline & 82,5 & 18 & 19 & 18 & 21 \\
\hline & 85 & 18 & 20 & 20 & 21 \\
\hline & 87,5 & 18 & 20 & 21 & 22 \\
\hline & 90 & 19 & 21 & 21 & 23 \\
\hline & 92,5 & 22 & 22 & 23 & 25 \\
\hline
\end{tabular}




\begin{tabular}{|c|c|c|c|c|c|}
\hline & 95 & 22 & 23 & 25 & 28 \\
\cline { 2 - 6 } & 97,5 & $\mathbf{2 2}$ & 25 & 30 & 30 \\
\cline { 2 - 6 } & 99 & $\mathbf{2 2}$ & 28 & 33 & 35 \\
\hline
\end{tabular}

Segundo os resultados da Tabela 1, tem-se que o modelo PRLSCP superestima o número de ambulâncias, ou seja, a quantidade de ambulâncias instaladas é excessiva para garantir o nível de confiança dado. O modelo BRLSCP precisou do mesmo número de ambulâncias que o modelo UBUL-M em apenas duas instâncias (veja cenário 01 para $\alpha=85 \%$ e cenário 02 para $\alpha=97,5 \%$ ), sendo que nas instâncias restantes o modelo UBUL-M demandou menos ambulâncias. A diferença chega a duas ambulâncias nos piores casos (veja o cenário 02 para $\alpha=82,5 \%$ até $\alpha=92,5 \%$ ).

O modelo QRLSCP, em quatorze instâncias, precisou do mesmo número de ambulâncias que o modelo PRLSCP e, em nove instâncias, o mesmo número de ambulâncias comparado ao modelo BRLSCP. Também obteve melhores resultados que o modelo PRLSCP para quatorze instâncias. Em relação ao modelo UBUL-M, no pior caso, houve uma diferença de treze ambulâncias (veja cenário 03 para $\alpha=99 \%$ ) e apenas um resultado do QRLSCP é melhor que o do modelo UBUL-M (veja cenário 01 para $\alpha=85 \%$ ).

Comparando o número total de ambulâncias, teve-se que o modelo UBUL-M demandou 408 ambulâncias, BRLSCP demandou 452, QRLSCP requisitou 469, e o modelo PRLSCP requisitou 490 unidades. Note que a diferença entre a quantidade total de ambulâncias do modelo UBUL-M com o modelo PRLSCP é de 82 unidades, ou 16,7\%. Em questões econômicas, esta diferença é bastante considerável.

A Tabela 2 mostra o tempo de otimização das duas implementações realizadas. Na implementação feita com o CPLEX e o GUROBI, limitou-se o tempo de execução em uma hora para cada instância (observe, por exemplo, o cenário 03 para $\alpha=92,5 \%$ até $\alpha=99 \%$ ). Dessa forma, em alguns casos, não foi encontrada a solução ótima. Assim, a solução e o tempo apresentados na tabela correspondem aos melhores valores encontrados durante a resolução da respectiva instância, dada uma variação no parâmetro $f$ de 1 até 3 .

Tabela 2: Tempos de otimização do CPLEX e do GUROBI.

\begin{tabular}{|c|c|c|c|c|c|}
\hline \multicolumn{6}{|c|}{ Implementação do Modelo UBUL-M } \\
\hline \multirow[b]{2}{*}{ Cenários } & \multirow[b]{2}{*}{$\alpha(\%)$} & \multicolumn{2}{|c|}{ ILOG CPLEX } & \multicolumn{2}{|c|}{ GUROBI OPTIMIZER } \\
\hline & & \# Ambulâncias & $\begin{array}{c}\text { Tempo de } \\
\text { otimização (s) }\end{array}$ & \# Ambulâncias & $\begin{array}{c}\text { Tempo de } \\
\text { otimização (s) }\end{array}$ \\
\hline \multirow{9}{*}{01} & 80 & 9 & 149,71 & 9 & 183,36 \\
\hline & 82,5 & 10 & $2.348,60$ & 10 & $2.241,35$ \\
\hline & 85 & 12 & 3.600 & 12 & 3600 \\
\hline & 87,5 & 12 & 0,81 & 12 & 0,61 \\
\hline & 90 & 12 & 1,49 & 12 & 1,90 \\
\hline & 92,5 & 12 & 1,34 & 12 & 0,67 \\
\hline & 95 & 12 & 1,59 & 12 & 0,68 \\
\hline & 97,5 & 12 & 2,61 & 12 & 8,07 \\
\hline & 99 & 17 & 3.600 & 17 & 3.600 \\
\hline \multirow{9}{*}{02} & 80 & 12 & 5,82 & 12 & 1,72 \\
\hline & 82,5 & 12 & 69,03 & 12 & 1,65 \\
\hline & 85 & 12 & 10,45 & 12 & 1,70 \\
\hline & 87,5 & 12 & 11,74 & 12 & 6,62 \\
\hline & 90 & 12 & 4,06 & 12 & 1,42 \\
\hline & 92,5 & 12 & 125,05 & 12 & 51,02 \\
\hline & 95 & 13 & 61,73 & 13 & 23,07 \\
\hline & 97,5 & 18 & 55,40 & 18 & 2,27 \\
\hline & 99 & 18 & 25,05 & 18 & 11,33 \\
\hline \multirow{6}{*}{03} & 80 & 18 & 104,23 & 18 & 14,01 \\
\hline & 82,5 & 18 & 108,49 & 18 & 30,37 \\
\hline & 85 & 18 & 107,83 & 18 & 29,80 \\
\hline & 87,5 & 18 & 108,25 & 18 & 29,85 \\
\hline & 90 & 19 & 234,93 & 19 & 86,34 \\
\hline & 92,5 & 22 & 3.600 & 22 & 3.600 \\
\hline
\end{tabular}




\begin{tabular}{|c|c|c|c|c|c|}
\hline & 95 & 23 & 3.600 & 22 & 3.600 \\
\cline { 2 - 5 } & 97,5 & 22 & 3.600 & 22 & 3.600 \\
\cline { 2 - 6 } & 99 & 38 & 3.600 & 22 & 3.600 \\
\hline
\end{tabular}

De acordo com a Tabela 2, nota-se que o tempo de otimização gasto pelo GUROBI é menor que o requerido pelo CPLEX. No GUROBI foi gasto um total de 6,75 horas, enquanto o CPLEX usou 6,98 horas de computação para resolver todas as instâncias.

Observa-se que para algumas instâncias o GUROBI apresentou tempos de otimização maiores do que o do CPLEX (veja cenário 01 para $\alpha=80 \%$ e $\alpha=97,5 \%$ ). Porém, na maioria dos casos, ocorreu o contrário. Na implementação usando GUROBI, o total de ambulâncias implantadas foi de 408, uma diferença razoável comparada a do CPLEX, que demandou 425 ambulâncias.

As Figuras 1 a 4 mostram a representação da solução para o cenário 02 dada a implementação usando CPLEX e GUROBI, respectivamente. Nestas figuras tem-se a quantidade de ambulâncias usadas e em quais estações (pontos vermelhos) foram instaladas.

Figura 1: Solução computada pelo CPLEX usando $\alpha=\mathbf{8 0} \%$.

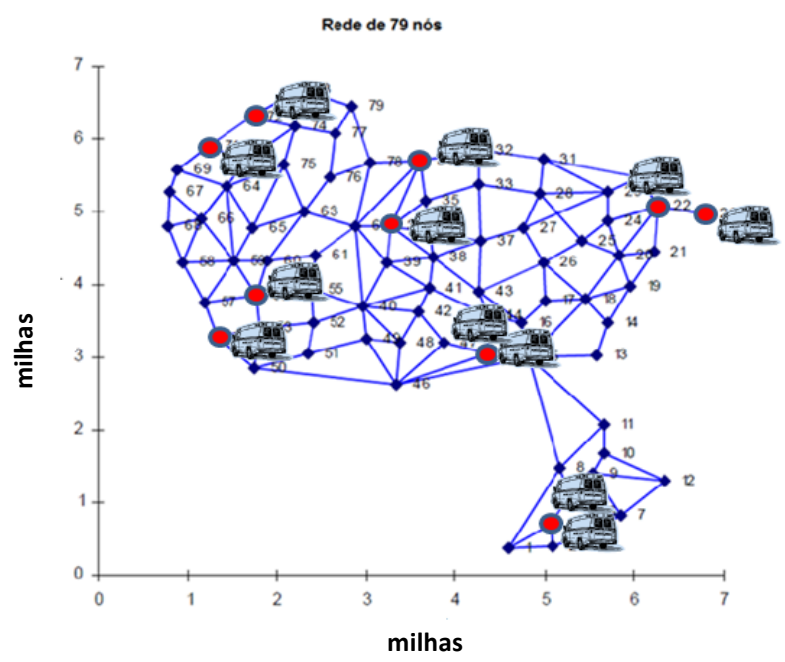

Figura 2: Solução computada pelo CPLEX usando $\alpha=97,5 \%$.

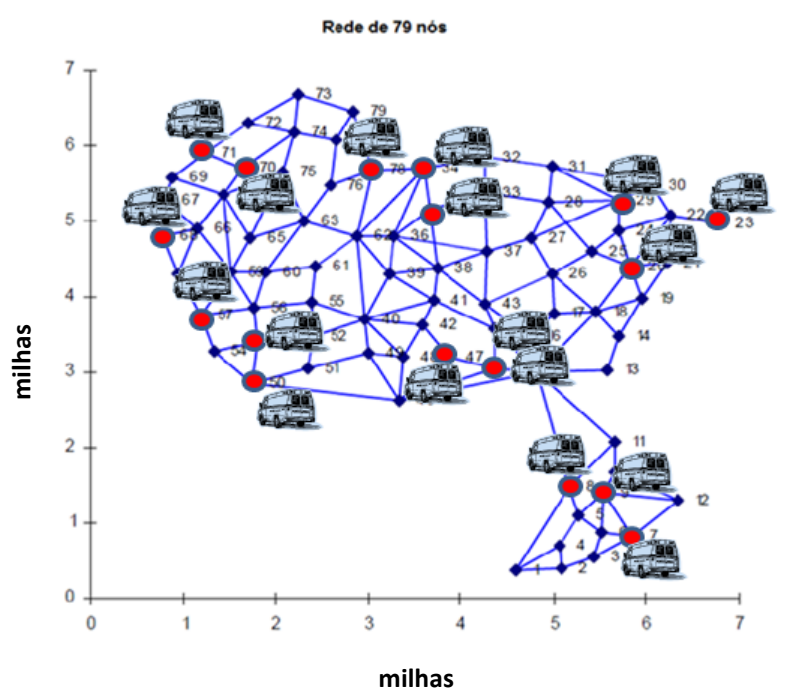

Figura 3: Solução computada pelo GUROBI usando $\alpha=\mathbf{8 0} \%$. 


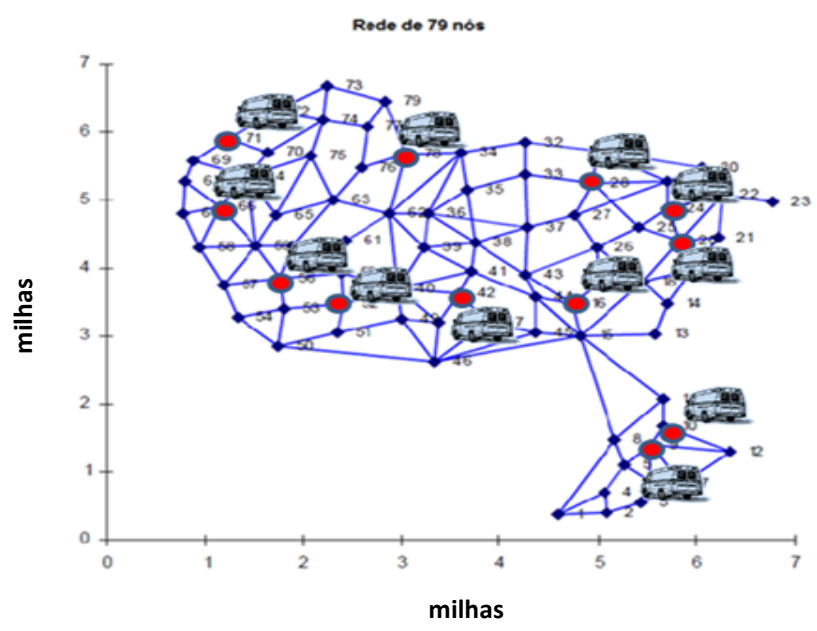

Figura 4: Solução computada pelo GUROBI usando $\alpha=97,5 \%$.

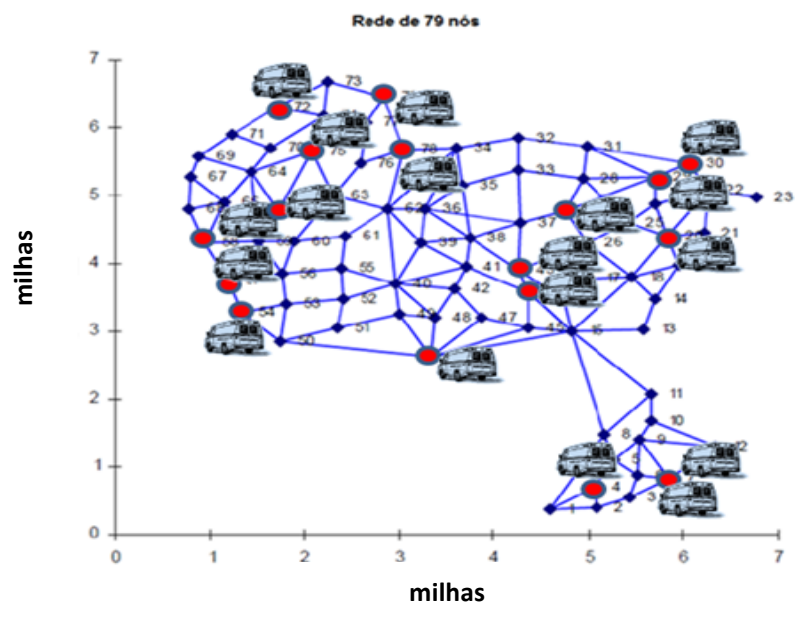

Observando as Figuras 1 a 4, percebe-se que a posição das estações e a disposição das ambulâncias mudam conforme o nível de confiabilidade. Isso acontece, pois a indisponibilidade das ambulâncias muda de acordo com o valor do parâmetro $\alpha$. Ou seja, para um nível de confiabilidade maior, estima-se um número maior de ambulâncias para atender todos os pontos de demanda que pertencem ao raio de cobertura das ambulâncias. A disposição das ambulâncias também muda de acordo com o solver, ou seja, existe mais de uma solução com o mesmo valor de função objetivo.

Ao comparar a solução das Figuras 1 e 2, nota-se claramente a mudança de posição das estações e o aumento de unidades. Na solução da Figura 1 são requeridas 12 ambulâncias, enquanto na Figura 2 são demandadas 18 ambulâncias. Também, pode-se observar que na Figura 4 foram instaladas duas ambulâncias no ponto de atendimento número sete. Neste caso, apenas esta estação foi necessária para atender a demanda, não sendo necessário abrir outra. Em termos práticos, abrir outra estação acarretaria em custos e não melhoraria o atendimento a população, dado o nível de confiança estabelecido.

\section{CONCLUSÕES}

O presente trabalho investiga o problema de localização de facilidades com aplicação em serviços de emergência. O foco do estudo foi um modelo matemático desenvolvido recentemente por Mohaymany et. al (2012), denominado UBUL, para determinar a localização de ambulâncias e estações (pontos de atendimento) com um limite de probabilidade de indisponibilidade e minimizando os custos de instalação. A partir deste modelo, fez-se a proposta de uma modificação, resultando no modelo UBUL-M, o qual foi comparado com a literatura. Similarmente ao UBUL, o UBUL-M retornou bons resultados para as instâncias de teste, impondo o uso de menos ambulâncias e garantindo o atendimento de toda a demanda.

Os resultados comparativos mostraram que o modelo UBUL-M abona a superestimação das ambulâncias, podendo conseguir um número menor de ambulâncias, satisfazendo toda a demanda e respeitando o nível mínimo de confiança. O modelo UBUL-M demandou a instalação de 425 ambulâncias no total, para a implementação feita no CPLEX, enquanto a do GUROBI solicitou 408 unidades. Por outro lado, dentre os modelos da literatura usados para comparação, o pior deles exigiu 490 unidades. Na administração pública (e privada) essa diferença é crucial e permite melhor uso dos recursos. O tempo total utilizando o CPLEX para o UBUL-M foi de 6,98 horas para resolver todas as 
instâncias, enquanto o do GUROBI limitou-se a 6,75 horas, dado um tempo de limite de uma hora para cada instância. Como neste estudo as decisões são de nível estratégico, que são de longo prazo, esse tempo de otimização é aceitável.

Uma análise do tempo de otimização pelos pacotes de otimização pode até influenciar o processo de tomada de decisão, de forma que uma proposta de trabalho futuro engloba o estudo de heurísticas. Apesar de não garantirem a solução ótima, uma boa heurística pode retornar soluções próximas, ou iguais a do modelo inteiro, porém gastando bem menos tempo computacional. Um estudo comparativo entre diferentes abordagens de resolução também seria algo interessante do ponto de vista teórico.

Por fím, é claro o reconhecimento e o estudo de problemas que permitam melhorar a área de saúde. Soluções para problemas desta natureza contribuem para um bem que transcende a área acadêmica.

\section{REFERÊNCIAS}

ARENAlES, A.; ARMENTANO, V. A.; MORABITO, R.; YANASSE, H. H. 2007. Pesquisa Operacional. Rio de Janeiro: Campus.

BATTA, R.; MANNUR, N. R.1990. Covering-Location Models for Emergency Situations That Require Multiple Response Units. Management Science, 36, 16-23.

BORRAS, F. 2000. Nuevos modelos probabilísticos de localización de servicios de emergencies. Tese de Doutorado, Departamento de Estadistica y Matematica aplicada, Universidad Miguel Hernandez de Elche, San Juan, Porto Rico.

BORRAS, F.; PASTOR, J. T. 2002. The ex-post evaluation of the minimum local reability level: Na enhanced probabilistic location set covering model. Annals of Operations Research, 111, 51-74.

CHURCH, R. L.; REVELLE, C. S. 1974. The maximal covering location problem. Papers of the Regional Science Association, 32, 101-118.

DASKIN, M. S.; STERN, E. H. 1981. A hierarchical objective set covering model for emergency medical service vehicle deployment. Transportation Science, 15, 137-152.

GALVAO, R. D.; MORABITO, R. 2008. Emergency service systems: The use of the hypercube queing model in the solution of probabilistic location problems. International Transactions in Operational Research, 15, 525-549.

GENDREAU, M.; LAPORTE, G.; SEMET, F. 1997. Solving an ambulance location model by Tabu search. Location Science, 5(2), 75-88.

GOLDBERG, J. B. 2004. Operations research models for the deployment of emergency services vehicles. EMS Management Journal, 1 (1), 20-39.

HAKIMI, S.L. 1964. Optimum location of switching centers and the absolute centers and the medians of a graph. Operations Research, 12 (3), 450-459.

HAKIMI, S. L. 1965. Optimum distribution of switching centers in a communication network. Operations Research, 13 (3), 462-475.

HOGAN, K.; REVELLE, C. S. 1986. Concepts and applications of backup coverage. Management Science, 34, $1434-$ 1444.

MOHAYMANY, A.S; BABAEI, M.; MOADI, S.; AMIRIPOUR, S. M. 2012. Linear Upper-Bound Unavailability Set Covering Models for Locating Ambulances: Application to Tehran Rural Roads, European Journal of Operational Research, 222 (1), 263-272.

NARASIMHAN, S.; PIRKUL, H.; SCHILLING, D. 1992. Capacitated emergency facility siting with multiple levels of backup. Annals of Operations Research, 40, 323-337.

NOGUEIRA, L. C. 2011. Um estudo para redução do tempo de resposta do SAMU de Belo Horizonte através da realocação das bases de operação. Dissertação de mestrado, Departamento de Engenharia de Produção, Universidade Federal de Minas Gerais, Belo Horizonte - MG, Brasil.

OWEN, S. H.; DASKIN, M. S. 1998. Strategic facility location: a review. European Journal of Operation Research, 111, 423-47.

PIRKUL, H.; SCHILLING, D. 1988. The siting of emergency service facilities with workload capacities and backup service. Management Science, 37 (7), 896-908.

REVELLE, C. S.; EISELT, H. A.; DASKIN, M. S. 2008. Bibliography for some fundamental problem categories in discrete location science. European Journal of Operational Research, 184, 817-848.

REVELLE, C. S.; HOGAN, K. 1989. The maximum availability location problem. Transportation Science, 23 (3), $192-$ 200.

SERRA, D. 1989. The pq-median problem: Location and Districting of hierarchical facilities. Ph. D. dissertation, Department of Geography and Environmental Engineering, John Hopkins University.

SILVA, V. L.; SUCENA, M. P. 2009. Localização de facilidades: Estudo de caso aplicado à escolha adequada de aeroporto para a minimização dos custos logísticos de distribuição de produtos farmacológicos. Disponível em: < http://cbtumetrorec.gov.br/monografia/2009/trabalhos/artigos/planejamento/2_246_AC.pdf>

TOREGAS, C. R. et al. 1971. The location of emergency service facilities. Operations Research, 19, 1363-1373.

\section{RESPONSABILIDADE AUTORAL}

Os autores são os únicos responsáveis pelo conteúdo deste trabalho. 


\title{
INVESTIGATION OF AN INTEGER FORMULATION FOR THE AMBULANCE AND STATIONS LOCATION PROBLEM
}

\author{
Raina Ribeiro Marques, raina-marques@hotmail.com \\ Thiago Alves de Queiroz, taq@ufg.br \\ Department of Mathematics, Federal University of Goiás / Campus Catalão, \\ Av. Dr. Lamartine Pinto de Avelar, 1120, Setor Universitário, 75704-020, Catalão-GO, Brazil.
}

\begin{abstract}
Facility location problems are useful when solving logistic problems, since they appear in public and private sectors, including the location of police stations, hospitals, schools, factories, warehouses, etc. This paper investigates an integer programming model that aims to minimize the cost of locate ambulances and service stations ensuring a minimum level of reliability to all services. The model under consideration was coded in the $C$ programming language and solved with the branch-and-cut algorithm available in the ILOG CPLEX and GUROBI OPTIMIZER libraries. Besides such model is compared with another ones from the literature, so results validate and show that it improves results from the literature in up to $16.7 \%$. We also present a comparative study about the run time required in each solver.
\end{abstract}

Keywords: Facility location problem, level of reliability, integer programming. 